\title{
LETTERS
}

DOI: $10.1017 / S 1041610204210912$

\section{P300 event-related potential in late-onset schizophrenia}

The P300 event-related potential (ERP) is a late positive waveform that is typically maximal at centroparietal midline sites $300 \mathrm{msec}$ post-stimulus in simple categorization tasks. Reduction in auditory P300 amplitude has been widely reported in young schizophrenic patients. The degree of amplitude reduction has been related to the degree of psychopathology, and seems relatively insensitive to changes in clinical status or to drug treatment. Auditory latency changes have not been so consistent, with some studies finding increased latencies but others not (Ford et al., 1994). Visual P300s appear to be more sensitive to changes in the clinical state of schizophrenic patients and are thus more variable than auditory P300 event-related potentials.

Nearly all ERP studies of schizophrenia have been conducted on persons who had a typical onset of the disorder in young adulthood. For several years there has been increasing interest in late-onset schizophrenia (defined as illness-onset between the ages of 40 and 60) (Howard et al., 2000). We were able to find only one ERP study investigating schizophrenia with onset of illness after age 40 . In that study (Olichney et al., 1998), 14 patients with late-onset schizophrenia did not have significantly smaller P300 auditory amplitudes than subjects from a normal control group, but patients with early-onset schizophrenia did. The purpose of this investigation was to further assess auditory and visual P300 ERPs in patients with late-onset schizophenia.

Subjects were selected from hospitalized patients who met DSM-IV criteria (American Psychiatric Association, 1994) for schizophrenia whose symptoms had begun after age 40 but before age 60 . Diagnoses were determined by a boardcertified psychiatrist. Symptoms had been present in each patient for 2 years or less at the time of the study, and in each case this was their first hospitalization. They had either never been treated with antipsychotic medication prior to admission, or if they had in the past, were not compliant and were off medication the month prior to admission. Each patient underwent history and physical examination and laboratory assessment, including complete blood count, chemistry survey, liver function studies, thyroid-stimulating hormone, urinalysis, and testing for syphilis. Potential subjects were excluded from the study if they had a medical illness or were on medications that could affect EEG, psychiatric illness other than schizophrenia, or significant histories of drug or alcohol abuse. Signed informed consent was obtained from each subject.

Prior to completion of the study, the only medication given was haloperidol, on an as-needed basis. Each subject underwent magnetic resonance imaging 
Table 1. Auditory and visual P300 latencies for subjects with late-onset schizophrenia and an age-matched control group

\begin{tabular}{|c|c|c|c|c|c|c|}
\hline & \multicolumn{2}{|c|}{$\begin{array}{l}\text { LATE-ONSET } \\
\text { SCHIZOPHRENIA } \\
\text { PATIENTS }\end{array}$} & \multicolumn{2}{|c|}{$\begin{array}{l}\text { AGE-MATCHED } \\
\text { CONTROL GROUP }\end{array}$} & \multirow[b]{2}{*}{$t$} & \multirow[b]{2}{*}{$p$} \\
\hline & MEAN & $\mathrm{SD}$ & MEAN & $\mathrm{SD}$ & & \\
\hline Auditory P300 latency & $360.9 \mathrm{msec}$ & 42.5 & $337.4 \mathrm{msec}$ & 14.0 & 1.66 & $0.114(\mathrm{~ns})$ \\
\hline Auditory P300 amplitude & $9.0 \mu \mathrm{V}$ & 2.38 & $10.3 \mu \mathrm{V}$ & 2.90 & -1.10 & $0.287(\mathrm{~ns})$ \\
\hline Visual P300 latency & $476.1 \mathrm{msec}$ & 34.7 & $462.9 \mathrm{msec}$ & 27.4 & 0.91 & $0.379(\mathrm{~ns})$ \\
\hline Visual P300 amplitude & $8.5 \mu \mathrm{V}$ & 1.49 & $9.9 \mu \mathrm{V}$ & 2.07 & -1.61 & $0.128(\mathrm{~ns})$ \\
\hline
\end{tabular}

(MRI) of the brain and recording of quantitative EEG and auditory and visual ERPs. Ten patients ( 1 male, 9 females; average age 52.8 years) completed the study. Data collected from the subjects were compared to a group of age-matched normal subjects (four males, six females, average age 56.0). MRI and quantitative EEG findings were largely unremarkable and have been reported (Reeves and Struve, 2003). That report also contains a detailed description of the individuals studied.

Auditory and visual P300 responses were obtained using an oddball paradigm, with the target stimulus occurring randomly in $15 \%$ of 300 stimulus presentations. For the auditory P300, the target tone was high-pitched $(2000 \mathrm{~Hz})$ as compared to the common tone $(1000 \mathrm{~Hz})$, delivered through earphones at $70 \mathrm{db}$. The visual P300 was based on a checkerboard square pattern reversal with target stimulus in large check size ( 15 checks across the screen) as opposed to small check sizes ( 30 checks across the screen) for the common stimulus. In both modalities, subjects counted the target stimuli, a 750 msec sweep was employed with a stimulus repetition rate of 0.9 per second, and high and low filters were set at 70 and $0.5 \mathrm{~Hz}$ respectively. P300 latency was measured at the most positive portion of the elicited peak. Amplitudes were measured from peak to peak (N2-P3).

Like most previous investigations of late-onset schizophrenia, this study demonstrates a female preponderance. Auditory P300 peaks were measurable in all ten subjects and visual P300 peaks in eight. In two subjects, visual P300 peaks could not be clearly identified. Table 1 shows the mean auditory and visual P300 latencies and amplitudes for the subjects with late-onset schizophrenia and the control group. Although mean latencies were longer and mean amplitudes were lower for the late-onset schizophrenia patients than for the control group, two tailed $t$-tests comparing these variables revealed no significant differences between the two groups.

The lack of significant ERP changes in these subjects with late-onset schizophrenia replicates and extends the previous study (Olichney et al., 1998), 
which showed that auditory P300 amplitudes were not decreased in such patients. P300 may possibly be a marker for a schizophrenia subtype with early onset and more severe information-processing deficits. It should be noted that our study represents findings relatively early in the course of late-onset schizophrenia. Further neurophysiological changes may occur as the disorder progresses. Additional research is needed in this area.

\title{
Acknowledgement
}

This research was supported in part by the South Central Mental Illness Research and Education Clinical Center (MIRECC).

\section{References}

American Psychiatric Association. (1994). Diagnostic and Statistical Manual of Mental Disorders (4th ed.) Washington, DC: American Psychiatric Association.

Ford, J. M. (1994). Schizophrenia: the broken P300 and beyond. Psychophysiology, 36, 667-682.

Howard, R., Rabins, P. V., Seeman, M. V. and Jeste, D. V. and the International Late-Onset Schizophrenia Group. (2000). Late-onset schizophrenia and very-late-onset schizophrenia-like psychosis: an international consensus. American fournal of Psychiatry, 157, $172-178$.

Olichney, J. M., Iragui, V. J., Kutas, M., Nowacki, R., Morris, S. and Jeste, D. V. (1998). Relationship between auditory P300 amplitude and age of onset of schizophrenia in older patients. Psychiatry Research, 79, 241-254.

Reeves, R. R. and Struve, F. A. (2003). Quantitative electroencephalography in late-onset schizophrenia. International Psychogeriatrics, 15, 273-278.

\author{
Roy R. ReEves, * Frederick A. STRUVE** AND JeFFERson D. PARKER*** \\ *Professor of Psychiatry and Neurology, University of Mississippi School of Medicine \\ and Chief of Mental Health, VA Medical Center, 1500 E. Woodrow Wilson Drive, Jackson, \\ MS 39216, U.S.A. \\ EMail: roy.reeves@med.va.gov \\ **Associate Research Scientist, Department of Psychiatry, Yale University School \\ of Medicine and VA Connecticut Health Care System. \\ ***A Assistant Professor of Psychiatry, University of Mississippi School of Medicine and Director \\ of Chemical Dependency Jackson (MS) VA Medical Center.
}

\section{DOI: $10.1017 / \$ 1041610204220919$}

\section{Charles-Bonnet syndrome and musical hallucination}

Both Charles-Bonnet syndrome and musical hallucinations are complex hallucinations (Menon et al., 2002; Ey, 1973). Raising awareness about the association between these two uncommon disorders is important. We would like to present two cases where this association is apparent. Consent for publication has been obtained. 
$\mathrm{MrX}$ is an 86-year-old man who was referred by his general practitioner to the psychiatric unit because he was having visual hallucinations. These were of men with vague faces, including two businessmen, and also children. They appeared in his left eye's lateral field and they did not speak. Mr X had a lateral field defect compatible with an old detached left retina for some time. In the absence of underlying brain disease, a diagnosis of Charles-Bonnet syndrome was made.

$\mathrm{Mr} \mathrm{X}$ also presented with musical hallucinations at night in his left ear, which had been deaf since he was 4 years old. He described these hallucinations as "the most beautiful music I have ever heard". It was a pure whistle, like opera and church music. Both psychiatric and neurological examinations were normal. There was no evidence of cognitive impairment. Detailed physical examination and investigations yielded normal results.

Mrs Y is an 86-year-old lady who was referred because of musical hallucinations. She heard the voice of the famous tenor Pavarotti singing opera and hymns unaccompanied. She did have some hearing impairment.

Her hallucinations were constant and overpowering and did not respond much to distraction. They were unilateral in the left ear. She responded to some extent to a small dose of olanzapine (2.5 $\mathrm{mg}$ daily).

A few months later, Mrs Y started to have visual hallucinations, but only in the left eye and its lateral visual field. In that area she saw a lady wearing a ballgown dancing. No abnormality with her eyes was reported. Charles-Bonnet syndrome was diagnosed. These visual hallucinations in her left peripheral visual field have disappeared after commencing anticonvulsant medication (carbamazepine $200 \mathrm{mg}$ twice daily).

A few months later, she began to show evidence of cognitive impairment, scoring 22/30 on the Mini-mental State Examination. Brain CT scan showed atrophic changes and small infarcts. A diagnosis of dementia was made. Both types of hallucinations disappeared completely when she moved to a nursing home.

Both visual and musical hallucinations are examples of the response of the sensory-deprived cerebral cortex. The corresponding cortical areas are not receiving enough stimulation and essentially make their own entertainment. Areas of the cortex that are redundant because of isolation, visual and hearing impairment, are prone to generate abnormal experiences (Berrios and Brook, 1985; Berrios, 1990; Menon et al., 2002). The association of these two cases of Charles-Bonnet syndrome and musical hallucination tends to support this, as does the disappearance of the hallucinations in the second case, once the lady was living in a nursing home, where there was a company and care, which reduced her isolation.

It is also possible that the syndrome can be an indication of early dementia (Pliskin et al., 1987). This is also evident in the second case. 
Clinicians should be aware of the association between Charles-Bonnet syndrome and musical hallucinations. Explanation and reassurance about the condition, together with reduction of isolation and an increase in stimulation are helpful to the patients. In all cases it is worth trying a small dose of atypical antipsychotic or anticonvulsant medication.

\section{References}

Berrios, G. E. (1990). Musical hallucinations: a historical and clinical study. British fournal of Psychiatry, 156, 188-194.

Berrios, G. E. and Brook, P. (1985). Visual hallucinations and sensory delusions in the elderly. British fournal of Psychiatry, 144, 662-664.

Ey, H. (1973). Traité des Hallucinations. Paris : Masson et Cie.

Menon, G. J., Rahman, I., Menon, S. J. and Dutton, G. N. L. (2003). Complex visual hallucinations in the visually impaired: the Charles-Bonnet Syndrome. Survey of Opthalmology, $48,58-72$.

Pliskin, N. H., Kiolbasa, T. A., Towle, V. I. et al. (1987) Charles-Bonnet syndrome. A study of 434 consecutive patients referred to a psychogeriatric unit. Family Practice, 4, 27-35.

ViCTOR M. AZIZ,* MAged NeSSIM** AND NiCHOLAS J. WARNER***

*Special Registrar in Old Age Psychiatry, Gwent Healthcare NHS Trust,

St Cadoc's Hospital, Caerleon, Newport, Gwent, South Wales, NP18 3XQ. U.K.

Email: victoraziz@doctors.org.uk

** Specialist Registrar in Ophalmology, Birmingham and Midland Eye Centre, U.K.

${ }^{* * *}$ Consultant Old Age Psychiatrist, Magnolia House, Summerlands, Yeovil, Somerset, U.K.

DOI: 10.1017/S1041610204230915

\section{Hospital admissions for acute onset of behavioral symptoms in demented patients: what do they want to say?}

Behavioral and psychological symptoms are a common manifestation of dementia (Grossberg, 2002). The identification of the cause that leads to behavioral disturbances is a critical step in determining an appropriate treatment strategy. We would like to report data regarding 148 demented patients ( $72.3 \%$ females) consecutively admitted during 2002 to the acute geriatric unit of the Civili Hospital in Brescia, Italy. The clinical reports were retrospectively reviewed in order to compare demented patients admitted for medical illnesses to ones admitted for acute onset of behavioral symptoms or delirium.

Fifty-eight patients were affected by Alzheimer Disease (AD), 49 by vascular dementia (VaD), 29 by mixed dementia $(\mathrm{AD}+\mathrm{VaD})$, and 12 by other types of dementia: Lewy Body dementia, dementia in alcohol abusers, in Parkinson Disease and frontotemporal dementia. The mean age was $82.3 \pm 9.3$ years, $24.4 \%$ lived alone, and the average number of diseases was $6.2 \pm 2.4$. While the majority of patients were admitted because of somatic diseases, 43 were 
admitted with a diagnosis of acute onset of one or more behavioral disturbances or delirium. The most frequent behavioral symptoms that caused hospital admission were: agitation (39.5\%), anorexia (35\%), depression/apathy (23\%), hallucinations and delusions (19\%), and aggression (18.5\%). Seven patients had delirium. During the hospital period, a somatic disease underlying the behavioral symptoms was identified in almost all of the 43 demented subjects. The most frequent medical illnesses were: urinary infections (35\%), adverse drug effects $(14 \%)$, pneumonia (11\%), vascular diseases $(9 \%)$, oral candidiasis $(7 \%)$, malignancy $(7 \%)$, new dementia cases $(5 \%)$, and uncontrolled diabetes $(5 \%)$. Only three patients had no diagnosis of somatic disease. Comparing the two groups of demented persons in univariate analysis, those with acute onset of behavioral symptoms were less disabled and had a lower pre-admission clinical dementia rating score (CDR) (Hughes et al., 1982) than those who were admitted for somatic illnesses. They did not significantly differ by age, gender, cohabitation, number of diseases and drugs used. In multivariate analysis, adjusted for age and gender, a lower CDR score was associated with being admitted for acute behavioral symptoms $(\mathrm{OR}=2.4,95 \% \mathrm{CI}=1.4-4.1)$.

Three major points can be deduced from these observations. First, persons affected by dementia might give evidence of medical illnesses through the acute onset of behavioral symptoms or delirium. Second, a large proportion of our patients developed behavioral disturbances because of somatic diseases easy to detect and to treat at home, such as urinary infections. Third, patients affected by milder dementia were more likely to be admitted for acute behavioral symptoms than those who were severe cases. We speculate that patients with better cognition are still able to show their discomfort and pain, developing behavioral symptoms. Behavioral symptoms increase resource utilization, often marking the transition between living in the community and institutionalization (Hemels et al., 2001). Even though antipsychotic drugs have a relatively high success rate (Edell and Tunis, 2001), they are not lacking in adverse effects and should not be used without a thorough assessment to search for underlying causes of behavioral change (Herrmann, 2001).

\section{References}

Edell, W. S. and Tunis, S. L. (2001). Antipsychotic treatment of behavioral and psychological symptoms of dementia in geropsychiatric inpatients. American fournal of Geriatric Psychiatry, 9, 289-297.

Grossberg, G. T. (2002). The ABC of Alzheimer's disease: behavioral symptoms and their treatment. International Psychogeriatrics, 14, 27-49.

Hemels, M. E., Lanctot, K. L., Iskedjian, M. and Einarson, T. R. (2001). Clinical and economic factors in the treatment of behavioural and psychological symptoms of dementia. Drugs and Aging, 18, 527-550. 
Herrmann, N. (2001). Recommendations for the management of behavioural and psychological symptoms of dementia. Canadian fournal of Neurological Sciences, 28, S96-S107.

Hughes, C. P., Berg, L., Danziger, W. L., Coben, L. A. and Martin, R. L. (1982). A new clinical scale for the staging of dementia. British fournal of Psychiatry, 140, 566-572.

\section{Alessandra Marengoni, antonio Paolo Calabrese}

AND STEFANIA Cossi

Geriatric Unit - I Medicina, Spedali Civili - Richiedei Foundation, Department of Medical and Surgery Sciences, University of Brescia: Piazzale Spedali Civili 125123 Brescia, Italy

Email: Alessandra.Marengoni@neurotec.ki.se

DOI: 10.1017/\$1041610204240911

\section{Cerebrovascular events after treatment of dementia patients with risperidone}

The behavioral and psychological symptoms of dementia (BPSD) often have a considerable negative impact on the everyday life of patients and their caregivers. Several non-pharmacological interventions such as training for caregivers or environmental adjustments have been suggested as treatment of BPSD (e.g. Burgio, 1996). Nevertheless, many of these patients will need psychopharmacological interventions, and antipsychotics have been shown to be the most effective class of psychotropics to treat such symptoms. Conventional antipsychotics markedly increase the risk for extrapyramidal side-effects, while atypical antipsychotics combine a high efficacy with a low rate of side-effects.

Two large double-blind, placebo-controlled trials have shown that the atypical antipsychotic risperidone has the capacity to reduce the frequency and severity of BPSD (De Deyn et al., 1999; Katz et al., 1999). In addition, these studies found that risperidone causes less severe extrapyramidal side-effects as compared to haloperidol, and was well tolerated overall. A third double-blind, placebocontrolled trial performed in Australia and New Zealand confirmed that risperidone reduces aggression, agitation and psychosis associated with dementia (Brodaty et al., 2003). But this study reported a high rate of cerebrovascular adverse events among those receiving risperidone. Five patients in the risperidone group $(3.0 \%)$ experienced stroke, and two of them died (1.2\%). In order to analyze the frequency of cerebrovascular adverse events among dementia patients treated with risperidone, the data of a survey conducted among Austrian primary care patients were used (Wancata, 2004).

Dementia patients over 60 years old living in the community and being treated routinely by primary care physicians for BPSD were included. Only patients who had not taken risperidone previously were eligible for this open-label prospective study. Exclusion criteria were the usual contraindications for treatment with risperidone. Data were collected by 203 general practitioners. Patients began treatment with a daily dose of $0.5 \mathrm{mg}$ risperidone for 3 days, and thereafter, the 
daily dose was adjusted as needed. Patients received risperidone for at least 6 weeks.

Since primary care physicians are not trained to use complex research instruments, a simple questionnaire was developed for this survey. It included a list of 13 possible BPSD symptoms which had to be assessed before risperidone treatment was started. After 6 weeks, these 13 BPSD symptoms were assessed again, and physicians made an overall judgment of how well risperidone was tolerated (excellent, satisfactory, not satisfactory). Physicians were asked to note all adverse events observed. Based on their clinical experience, the physicians assessed each observed adverse event with respect to a possible relation to risperidone intake. Adverse events were assessed after 2 and 6 weeks. (Following the Austrian Drug Law, an Ethical Approval was not required for this survey, where patients received risperidone as part of their routine treatment.)

Nine hundred and thirty-eight elderly persons (69.1\% male) with a mean age of 81.2 years (SD 7.9) participated in the study. According to the physicians' assessments, $36.6 \%$ of the patients were judged to have severe and $49.3 \%$ moderate BPSD. Only $14.1 \%$ suffered from mild BPSD. The majority of BPSD symptoms $(72 \%)$ improved during treatment with risperidone.

One patient $(0.1 \%)$ experienced a stroke with a lethal outcome about two weeks after the start of risperidone treatment. This 90-year old man received $1 \mathrm{mg}$ risperidone per day. Concomitant diseases included prostatica adenoma, struma, and renal insufficiency. Besides risperidone, he had received treatment with piracetam, gingko biloba, metildigoxin and doxycyclin. According to the treating physician, his death was not related to the treatment with risperidone.

Another two patients $(0.2 \%)$ suffered a temporary weakness in one of their legs. In both cases the treating physicians did not classify these symptoms as a transient ischemic attack (TIA), but it cannot be excluded that there was a causal relationship with a cerebrovascular event. One of the patients had had a stroke in the past, and the other had polyneuropathy and diabetes. The first patient took $1 \mathrm{mg}$ risperidone per day and the second $0.5 \mathrm{mg}$ per day. The physician of the second patient judged that there could be a "possible" relationship of the temporary weakness to risperidone, while the physician of the first patient did not find such a association. Both physicians did not terminate risperidone and did not initiate hospitalization. At the end of the 6 weeks, both physicians reported that the tolerability of risperidone was "satisfactory".

Overall, the results of this open-label study show a very low rate of cerebrovascular events among dementia patients treated with risperidone. This rate is markedly lower than that reported from the Australian and New Zealand study (Brodaty et al., 2003). It needs to be taken into account that doubleblind, placebo-controlled trials have certain limitations because they are obliged to use very strict exclusion criteria, which result in highly selected samples. 
Since patients suffering from dementia are usually treated as outpatients by primary care physicians, the present study was performed in a more "real life" environment than that of double-blind, placebo-controlled trials.

Overall, in the present study, adverse events were reported rarely $(7.4 \%)$, while in a randomized trial (Katz et al., 1999), about $80 \%$ of patients receiving risperidone had an adverse event. When interpreting these results, two points have to be considered: the questionnaire developed for this study and used for data collection was not tested for validity and reliability, and the primary care physicians had not been trained in the assessment of side-effects of antipsychotics. Thus, we cannot rule out that in the present study some adverse events were not detected. It seems however very unlikely that physicians would not notice and report severe adverse events like a stroke or a TIA.

As well as assessing single adverse events, the physicians in the present survey evaluated the overall treatment tolerability and reported that in more than $80 \%$ of cases, the tolerability was "excellent". Thus, despite the potential limitations mentioned above, the present results indicate that cerebrovascular adverse events occur very rarely among dementia patients treated with risperidone in primary care.

\section{References}

Brodaty, H., et al. (2003). A randomized placebo-controlled trial of risperidone for the treatment of aggression, agitation, and psychosis in dementia. Fournal of Clinical Psychiatry, 64, 134-143.

Burgio, L. (1996). Interventions for the behavioral complications of Alzheimer's Disease: behavioral approaches. International Psychogeriatrics, 8 (Suppl. 1), 45-52.

De Deyn, P. P., et al. (1999). A randomized trial of risperidone, placebo, and haloperidol for behavioral symptoms of dementia. Neurology, 53, 946-955.

Katz, I. R., Jeste, D. V., Mintzer, J. E., Clyde, C., Napolitano, J. and Brecher, M. (1999). Comparison of risperidone and placebo for psychosis and behavioral disturbances associated with dementia: a randomised, double-blind trial. fournal of Clinical Psychiatry, 60, 107-115.

Wancata, J. (2004). Efficacy of risperidone for treating patients with behavioral and psychological symptoms of dementia. International Psychogeriatrics, 16, 107-115.

JOHANNES WANCATA

Medical University of Vienna, Department of Psychiatry, Währinger Gürtel 18-20, 1090

Vienna, Austria.

Email: johannes.wancata@meduniwien.ac.at

\section{Conflict of interest}

The author did not receive financial support for this study. Data collection and data entry was funded by Janssen-Cilag. The author has received speaker's honoraria from Janssen-Cilag. 